\title{
Generals of Iowa Civil War Regiments
}

\author{
by Richard Hellie
}

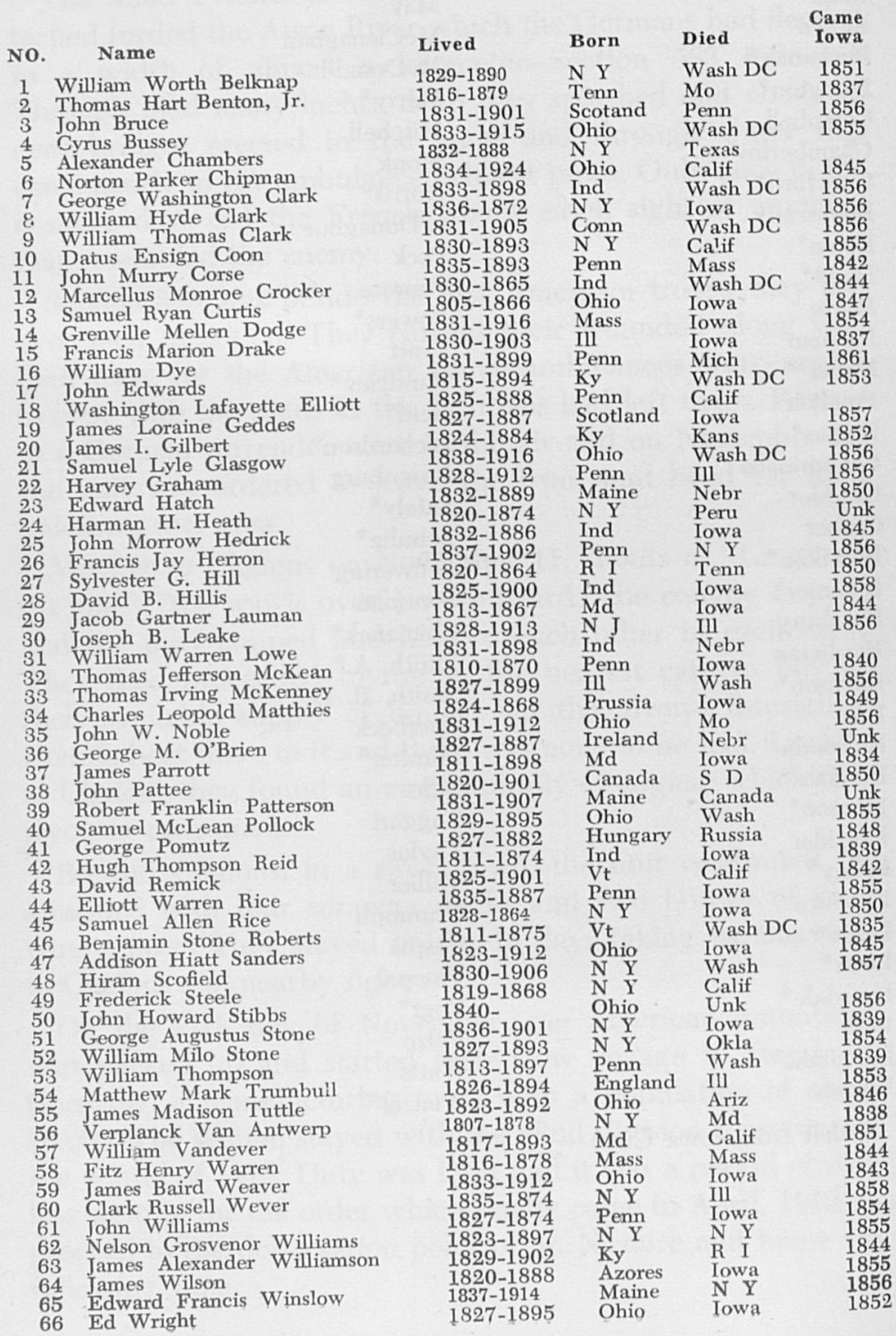




\section{Code}

Bank-banking

Cb-Carpetbagger

Ed-Education

Eng-Engineering

$\mathrm{F}$, Farm.-farming

G, Gov.-government

J, Journ.-journalism

L, Law.-law

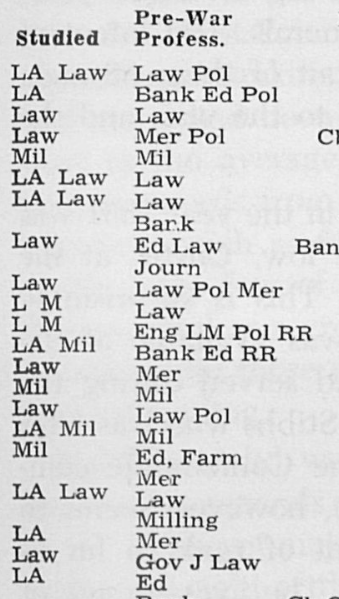

LA Bank

Med Med Mer

LA Law Bank Mer

Mil Law Law Pol

Mil Eng F Pol RR

Med Med

Mil Mer

LA Law G L Mer

Eng

M Pol Mer

Carpenter

Law Law Po

Mil Mil

LA Law Law

LA Law Law

LA Law Law Pol

L M Eng Mil RR

LA Z Ed J

LA Law Ed Law

Mil

Mil

LA

Mer

Law

J Law

Law

L M

Law

LA

Law

J Law Pol

Ed Law Pol

Farm Pol Mer

Gov J L Pol

$\mathrm{J}$ L Pol

G J Pol Mer

J Law

Broker

Law Farm

Mil Farm Mer

LA Law Law Pol

Farm Mer

RR Mer

Ed F Pol
LA-Liberal Arts

Med-medicine

Mer-merchandising

M, Mil.-military

Min-mining

Mfg-manufacturing

Pol-politics

RR-railroad

Post-War
Profess.
Gov Pol
Gov Pol
Law
Pal Mer
Mil
Gov Pol
Gov

Last

Entered Highest Rank

Profess.

Army as

obtained

No.

Bank Pol

Gov Law Pol

Bank RR

Gov Law

Gov Law

Mil Pol

Gov Law

Gov

Bark

$\mathrm{Pol} \mathrm{Cb}$

Gov $\mathrm{Po}$

Gov Pol RR

Deceased Deceased

Gov

$\mathrm{RR}$

Pol RR

Law

Mil

F G J Mil

Cb Gov

Mil Bank

Ed

Mer Mer

Law Pol

Milling

Mil Mil

Gov Tramp

Gov FG J Pol RR

Cb G Law Pol

Mfg

Deceased Deceased

Med Gov Med

Sick Deceased

Pol Law Gov Law

Mfg Min

Pol Farm Pol

Gov Mer

Mer Mer Pol

Law Gov Law

RR Gov

Mil

Law Law

Gov Gov

RR RR

Law Law

Deceased Deceased

Mil GL Mfg

Gov J Mer

Law Law

Mil Gov Mil

Goy Gov

Bank Gov Mer

Goy Pol G L Pol

$\mathrm{Cb} \mathrm{J}$ Mil

Law Gov Journ

Farm Mfg Min Pol

Mil Mil

RR GP RR

Pol Gov Pol

Law Pol Gov Pol

Bank Constr

Law Journ

Gov

Cb Law GP RR

Farm Bank

RR Gov RR

Farm Pol Gov Pol
Maj Brev Maj Gen 1

Col Brev Brig Gen 2

Capt Brig Gen

Col Brev Gen 4

Pri Brev Brig Gen 6

1st Lt Brev Brig Gen 7

1st Lt Brev Brig Gen 8

Adjt Brev Brig Gen 9

Capt Brev Brig Gen 10

Maj Brev MajGen 11

Capt Brig Gen 12

Col MajGen 13

Col Maj Gen 14

Lt Col Brev Brig Gen 15

Col Brev Brig Gen 16

Col Brev Brig Gen 17

Col Brev Maj Gen 18

Capt Brig Gen 19

Col Brev Maj Gen 20

1st Lt Brev Maj Gen 21

1st Lt Brev Brig Gen 22

Maj Brev Maj Gen 123

Maj Brev Maj Gen 24

1st Lt Brev Brig Gen. 25

Capt Maj Gen 26

Capt Brev Brig Gen 27

Lt Col Brev Brig Gen 28

Col Brev Maj Gen 29

Capt Brev Brig Gen 30

Col Brev Brig Gen 31

Paym Brev Maj Gen 32

1st Lt Brev Brig Gen 33

Capt Brig Gen 34

Adjt Brev Brig Gen 35

Capt Brev Brig Gen 37

Capt Brev Brig Gen 38

2nd Lt Brev Brig Gen 39

Lt Col Brev Brig Gen 40

1st Lt Brev Brig Gen 41

Col Brig Gen 42

Capt Brev Brig Gen 43

Pri Brev Maj Gen 44

Col Brig Gen 45

Maj Brev Maj Gen 46

Lt Col Brig Gen 47

Pri Brev Brig Gen 48

Col Maj Gen 49

Capt Brev Brig Gen 50

1st Lt Brev Brig Gen 51

Capt Brev Brig Gen 52

Capt Brev Brig Gen 53

Capt Brev Brig Gen 54

Lt Col Brig Gen 55

Maj Brev Brig Gen 56

Col Brev Maj Gen 57

Col Brev Maj Gen 58

1st Lt Brev Brig Gen 59

Capt Brev Brig Gen 60

Capt Brev Brig Gen 61

Adjt Brev Maj Gen 63

1st Lt Brev Brig Gen 64

Capt Brev Brig Gen 65

Maj Brev Brig Gen 66
Col Brev Brig Gen 5

Maj Brev Brig Gen 36

Col Brig Gen 62 
Copyright of Annals of Iowa is the property of State of Iowa, by \& through the State Historical Society of Iowa and its content may not be copied or emailed to multiple sites or posted to a listserv without the copyright holder's express written permission. However, users may print, download, or email articles for individual use. 